\title{
A Maximum Weighted Likelihood Approach to Simultaneous Model Selection and Feature Weighting in Gaussian Mixture
}

\author{
Yiu-ming Cheung and Hong Zeng \\ Department of Computer Science, Hong Kong Baptist University, Hong Kong \\ \{ymc, hzeng\}@comp.hkbu.edu.hk
}

\begin{abstract}
This paper is to identify the clustering structure and the relevant features automatically and simultaneously in the context of Gaussian mixture model. We perform this task by introducing two sets of weight functions under the recently proposed Maximum Weighted Likelihood (MWL) learning framework. One set is to reward the significance of each component in the mixture, and the other one is to discriminate the relevance of each feature to the cluster structure. The experiments on both the synthetic and real-world data show the efficacy of the proposed algorithm.
\end{abstract}

\section{Introduction}

The finite mixture model has provided a formal approach to address the clustering problems. In this unsupervised domain, there are two key issues. One is the determination of an appropriate number of components (also called number of clusters or model order interchangeably) in a mixture model. In general, the true clustering structure may not be well described with too few components, whereas the estimated model may "over-fit" the data if it uses too many components. The other issue is how to identify the relevance of observation features with respect to the clustering structure. From the practical viewpoint, some features may not be so important, or even be irrelevant, to the clustering structure. Their participation in the clustering process will prevent a clustering algorithm from finding an appropriate partition. Hence, it is necessary to discriminate the relevance of each feature with respect to the clustering structure in the clustering analysis.

Clearly, the above two issues are closely related. However, most of the existing approaches deal with these two issues separately or sequentially. Some methods typically choose the most influential features prior to a clustering algorithm, e.g., see 12 . Although the success of their algorithms has been demonstrated in their application domains, these pre-selected features may not be necessarily suitable to the clustering algorithm that will be ultimately employed. Moreover, some approaches 34] wrap the clustering algorithms in an outer layer to evaluate the candidate feature subsets. The performance of such a method is superior to that of the previous approaches, but their search strategies of generating the feature 
subset candidates are prone to find a local maxima. Recently, it has been believed that the above two issues should be jointly optimized in a single learning paradigm. A typical example is the work of [5], which introduces the concept of feature saliency to measure the relevance of each feature to the clustering structure, as the feature weight. It then heuristically integrates the Minimum Message Length (MML) criterion into the likelihood, and optimizes this penalized likelihood using a modified Expectation-Maximization (EM) algorithm to obtain the feature weights and the clustering results. Nevertheless, the penalty terms given by the MML criterion are static and fixed for all components at each EM iteration. As a result, they may not be robust enough under a certain environment, in which it is more desirable to implement a dynamic and embedded scheme to control the model complexity.

In this paper, we propose such an approach to Gaussian mixture clustering by formulating the above two issues into a single Maximum Weighted Likelihood (MWL) optimization function [6]. We introduce two sets of weight functions to address these two issues, respectively. Consequently, both the model selection and feature weighting are performed automatically and simultaneously. The experiments on both the synthetic and real-world data have shown the efficacy of the proposed algorithm.

\section{The MWL Learning Framework}

Suppose $N$ i.i.d. observations, denoted as $\mathbf{x}_{\mathbf{1}}, \mathbf{x}_{\mathbf{2}}, \ldots, \mathbf{x}_{\mathbf{N}}$, come from the following mixture model:

$$
p\left(\mathbf{x}_{t} \mid \Theta^{*}\right)=\sum_{j=1}^{k^{*}} \alpha_{j}^{*} p\left(\mathbf{x}_{t} \mid \theta_{j}^{*}\right)
$$

with

$$
\sum_{j=1}^{k^{*}} \alpha_{j}^{*}=1 \quad \text { and } \quad \forall 1 \leq j \leq k^{*}, \quad \alpha_{j}^{*}>0,
$$

where each observation $\mathbf{x}_{\mathbf{t}}(1 \leq t \leq N)$ is a column vector of d-dimensional features, i.e. $\mathbf{x}_{\mathbf{t}}=\left[x_{1 t}, \ldots, x_{d t}\right]^{T}$, and $\Theta^{*}=\left\{\alpha_{j}^{*}, \theta_{j}^{*}\right\}_{j=1}^{k^{*}}$. Furthermore, $\theta_{j}^{*}$ denotes the parameter set of the $j$ th probability density function (pdf) $p\left(\mathbf{x}_{t} \mid \theta_{j}^{*}\right)$ in the mixture model, $k^{*}$ is the true cluster number, and $\alpha_{j}^{*}$ is the mixing proportion of the $j$ th component in the mixture. $\Theta^{*}$ is estimated from these $N$ observations by:

$$
\hat{\Theta}_{M L}=\arg \max _{\Theta}\left\{\log p\left(\mathbf{X}_{\mathbf{N}} \mid \Theta\right)\right\}
$$

where $\mathbf{X}_{\mathbf{N}}=\left\{\mathbf{x}_{\mathbf{1}}, \mathbf{x}_{\mathbf{2}}, \ldots, \mathbf{x}_{\mathbf{N}}\right\}$, and $\hat{\Theta}_{M L}=\left\{\alpha_{j}, \theta_{j}\right\}_{j=1}^{k}$ is a maximum likelihood (ML) estimate of $\Theta^{*}$.

When the number of components $k$ is known, the ML estimate in (2) could be obtained by the Expectation-Maximization (EM) algorithm. However, from the practical viewpoint, it is difficult or even impossible to know the number 
of components in advance. Recently, a promising approach called Rival Penalized Expectation-Maximization (RPEM for short) [6], where the model order is determined automatically and simultaneously with the parameter estimation, has been developed under the MWL learning framework. The main idea of the MWL framework is to introduce unequal weights in general into the conventional maximum likelihood, which actually provides a new promising way for regularization so that the weighted likelihood does not increase monotonically over the candidate model complexity. Specifically, the weighted likelihood is given bellow:

$$
\begin{aligned}
Q\left(\Theta, \mathbf{X}_{N}\right) & =\frac{1}{N} \sum_{t=1}^{N} \log p\left(\mathbf{x}_{t} \mid \Theta\right)=\frac{1}{N \zeta} \sum_{t=1}^{N} \sum_{j=1}^{k} g\left(j \mid \mathbf{x}_{t}, \Theta\right) \log p\left(\mathbf{x}_{t} \mid \Theta\right) \\
& =\frac{1}{N \zeta} \sum_{t=1}^{N} \mathcal{M}\left(\Theta, \mathbf{x}_{t}\right) \\
\mathcal{M}\left(\Theta, \mathbf{x}_{t}\right) & =\sum_{j=1}^{k} g\left(j \mid \mathbf{x}_{t}, \Theta\right) \log \left[\alpha_{j} p\left(\mathbf{x}_{t} \mid \theta_{j}\right)\right]-\sum_{j=1}^{k} g\left(j \mid \mathbf{x}_{t}, \Theta\right) \log h\left(j \mid \mathbf{x}_{t}, \Theta\right)
\end{aligned}
$$

where $h\left(j \mid \mathbf{x}_{t}, \Theta\right)=\frac{\alpha_{j} p\left(\mathbf{x}_{t} \mid \theta_{j}\right)}{p\left(\mathbf{x}_{t} \mid \Theta\right)}$ is the posterior probability that $\mathbf{x}_{t}$ belongs to the $j$ th component in the mixture, $k$ is an estimate of $k^{*}$ with $k \geq k^{*}$, and $\zeta$ is a constant. The $g\left(j \mid \mathbf{x}_{t}, \Theta\right)$ 's are the weight functions, satisfying the constraints:

$$
\forall t, j, \quad \sum_{j=1}^{k} g\left(j \mid \mathbf{x}_{t}, \Theta\right)=\zeta ; \lim _{h\left(j \mid \mathbf{x}_{t}, \Theta\right) \rightarrow 0} g\left(j \mid \mathbf{x}_{t}, \Theta\right) \log h\left(j \mid \mathbf{x}_{t}, \Theta\right)=0 .
$$

In the RPEM algorithm of [6], the weight functions are constructed as:

$$
g\left(j \mid \mathbf{x}_{t}, \Theta\right)=\left(1+\varepsilon_{t}\right) I\left(j \mid \mathbf{x}_{t}, \Theta\right)-\varepsilon_{t} h\left(j \mid \mathbf{x}_{t}, \Theta\right)
$$

with

$$
I(j \mid \mathbf{x}, \Theta)=\left\{\begin{array}{l}
1 \text { if } j=c \equiv \arg \max _{1 \leq i \leq k} h(i \mid \mathbf{x}, \Theta) ; \\
0 \text { otherwise. }
\end{array}\right.
$$

where $\varepsilon_{t}$ is a small positive quantity. Under this weight construction, given an observation $\mathbf{x}_{t}$, a positive weight $g\left(c \mid \mathbf{x}_{t}, \Theta\right)$ is assigned to the log-likelihood of the winning component, i.e., the component with the maximum value of $h\left(j \mid \mathbf{x}_{t}, \Theta\right)$, so that it is updated to adapt to $\mathbf{x}_{t}$, meanwhile all rival components are penalized with a negative weight. This intrinsic rival penalization mechanism of the RPEM makes the genuine clusters survive, whereas the "pseudo-clusters" gradually vanish. The updating details of $\hat{\Theta}_{M W L}=\arg \max _{\Theta}\left\{Q\left(\Theta, \mathbf{X}_{N}\right)\right\}$ can be found in [6].

The numerical results have shown its outstanding performance on both of synthetic and real-life data [6], where all features are equally useful in clustering process. Nevertheless, analogous to the most of the existing clustering algorithms, the performance of the RPEM may deteriorate provided that there exist some irrelevant features in feature vectors. In the following, we will therefore perform the feature relevancy analysis and further extend the RPEM accordingly within the MWL framework. 


\section{Simultaneous Clustering and Feature Weighting}

To discriminate the importance of each feature in the cluster structure, we utilize the concept of feature saliency defined in [5] as our feature weight (i.e., $w_{l}, 0 \leq$ $\left.w_{l} \leq 1, \forall 1 \leq l \leq d\right)$ : the $l$ th feature is relevant with a probability $w_{l}$ that the feature's pdf is dependent of the pdf's of components in the mixture. For those features whose values are distributed among all clusters, we regard its distribution as a common one. We suppose the features are independent of each other, then the pdf of a more general mixture model can be written below as in [5]:

$$
\begin{aligned}
p\left(\mathbf{x}_{t} \mid \Theta\right) & =\sum_{j=1}^{k} \alpha_{j} \prod_{l=1}^{d} p\left(x_{l t} \mid \Phi\right) \\
& =\sum_{j=1}^{k} \alpha_{j} \prod_{l=1}^{d}\left[w_{l} p\left(x_{l t} \mid \theta_{l j}\right)+\left(1-w_{l}\right) q\left(x_{l t} \mid \lambda_{l}\right)\right]
\end{aligned}
$$

where $p\left(x_{l t} \mid \theta_{l j}\right)=\mathcal{N}\left(x_{l, t} \mid m_{l j}, s_{l j}^{2}\right)$ denotes a Gaussian density function of the relevant feature $x_{l t}$ with the mean $m_{l j}$, and the variance $s_{l j}^{2} ; q\left(x_{l t} \mid \lambda_{l}\right)$ is the common distribution of the irrelevant feature. In this paper, we shall limit it to be a Gaussian as well for a general purpose, i.e., $q\left(x_{l t} \mid \lambda_{l}\right)=\mathcal{N}\left(x_{l t} \mid c m_{l}, c s_{l}^{2}\right)$. Subsequently, the full parameter set of the general Gaussian mixture model is redefined as $\Theta=\left\{\left\{\alpha_{j}\right\}_{j=1}^{k}, \Phi\right\}$ and $\Phi=\left\{\left\{\theta_{l j}\right\}_{l=1, j=1}^{d, k},\left\{w_{l}\right\}_{l=1}^{d},\left\{\lambda_{l}\right\}_{l=1}^{d}\right\}$. Note that

$$
p\left(x_{l t} \mid \Phi\right)=w_{l} p\left(x_{l t} \mid \theta_{l j}\right)+\left(1-w_{l}\right) q\left(x_{l t} \mid \lambda_{l}\right)
$$

is a linear mixture of two possible densities for each feature, and the feature weight $w_{l}$ acts as a regulator to determine which distribution is more appropriate to describe the feature. A new perspective is to regard this form as a lower level Gaussian mixture, which resembles the higher level Gaussian mixture on which the weights of the genuine clusters are estimated. Hence, the feature weight $w_{l}$ can be considered as the counterpart of component weight $\alpha_{j}$. Subsequently, a similar rewarding and penalizing scheme can be embedded into the likelihood function of (3) for this lower level mixture. To this end, we re-write (3) as:

$$
\tilde{Q}\left(\Theta, \mathbf{X}_{N}\right)=\frac{1}{N} \sum_{t=1}^{N} \log p\left(\mathbf{x}_{t} \mid \Theta\right)=\frac{1}{N \zeta} \sum_{t=1}^{N} \tilde{\mathcal{M}}\left(\Theta, \mathbf{x}_{t}\right) .
$$

To control the complexity of the model to be estimated, we introduce two sets of weight functions, i.e. $\tilde{g}\left(. \mid \mathbf{x}_{t}, \Theta\right)$ and $\tilde{f}\left(. \mid x_{l t}, \Phi\right)$, into the log-likelihood for the components in the higher level and lower level mixtures, respectively. Altogether, by inserting the following formulas:

$$
p\left(\mathbf{x}_{t} \mid \Theta\right)=\frac{\alpha_{j} p\left(\mathbf{x}_{t} \mid \Phi\right)}{\tilde{h}\left(j \mid \mathbf{x}_{t}, \Theta\right)} ; p\left(x_{l t} \mid \Phi\right)=\frac{w_{l} p\left(x_{l t} \mid \theta_{l j}\right)}{h^{\prime}\left(1 \mid x_{l t}, \Phi\right)}=\frac{\left(1-w_{l}\right) q\left(x_{l t} \mid \lambda_{l}\right)}{h^{\prime}\left(0 \mid x_{l t}, \Phi\right)},
$$

into (9), we obtain the weighted log-likelihood for the mixture model as follows: 


$$
\begin{aligned}
& \tilde{\mathcal{M}}\left(\Theta, \mathbf{x}_{t}\right)=\sum_{j=1}^{k} \tilde{g}\left(j \mid \mathbf{x}_{t}, \Theta\right) \log \alpha_{j}+ \\
& \sum_{j=1}^{k} \sum_{l=1}^{d} \tilde{g}\left(j \mid \mathbf{x}_{t}, \Theta\right)\left\{\tilde{f}\left(1 \mid x_{l t}, \Phi\right) \log \left[w_{l} p\left(x_{l t} \mid \theta_{l j}\right)\right]+\tilde{f}\left(0 \mid x_{l t}, \Phi\right) \log \left[\left(1-w_{l}\right) q\left(x_{l t} \mid \lambda_{l}\right)\right]\right\}- \\
& \sum_{j=1}^{k} \sum_{l=1}^{d} \tilde{g}\left(j \mid \mathbf{x}_{t}, \Theta\right) \tilde{f}\left(1 \mid x_{l t}, \Phi\right) \log h^{\prime}\left(1 \mid x_{l t}, \Phi\right)-\sum_{j=1}^{k} \sum_{l=1}^{d} \tilde{g}\left(j \mid \mathbf{x}_{t}, \Theta\right) \tilde{f}\left(0 \mid x_{l t}, \Phi\right) \log h^{\prime}\left(0 \mid x_{l t}, \Phi\right) \\
& -\sum_{j=1}^{k} \tilde{g}\left(j \mid \mathbf{x}_{t}, \Theta\right) \log \tilde{h}\left(j \mid \mathbf{x}_{t}, \Theta\right)
\end{aligned}
$$

where

$$
\begin{gathered}
\tilde{h}\left(j \mid \mathbf{x}_{t}, \Theta\right)=\frac{\alpha_{j} p\left(\mathbf{x}_{t} \mid \Phi\right)}{p\left(\mathbf{x}_{t} \mid \Theta\right)}=\frac{\alpha_{j} \prod_{l=1}^{d}\left[w_{l} p\left(x_{l t} \mid \theta_{l j}\right)+\left(1-w_{l}\right) q\left(x_{l t} \mid \lambda_{l}\right)\right]}{\sum_{i=1}^{k} \alpha_{i} \prod_{l=1}^{d}\left[w_{l} p\left(x_{l t} \mid \theta_{l i}\right)+\left(1-w_{l}\right) q\left(x_{l t} \mid \lambda_{l}\right)\right]}, \\
h^{\prime}\left(1 \mid x_{l t}, \Phi\right)=\frac{w_{l} p\left(x_{l t} \mid \theta_{l j}\right)}{w_{l} p\left(x_{l t} \mid \theta_{l j}\right)+\left(1-w_{l}\right) q\left(x_{l t} \mid \lambda_{l}\right)}, h^{\prime}\left(0 \mid x_{l t}, \Phi\right)=1-h^{\prime}\left(1 \mid x_{l t}, \Phi\right) .
\end{gathered}
$$

$\tilde{h}\left(j \mid \mathbf{x}_{t}, \Theta\right)$ indicates the probability that some features in the data points come from the $j t h$ density component in the subspace. $h^{\prime}\left(1 \mid x_{l t}, \Phi\right)$ represents the posterior probability that the $l$ th feature conforms to the mixture model. That is, it reflects the prediction for the relevance of the $l$ th feature to the clustering structure.

We design the weight functions for the higher level mixture as follows:

$$
\tilde{g}\left(j \mid \mathbf{x}_{t}, \Theta^{o l d}\right)=I\left(j \mid \mathbf{x}_{t}, \Theta\right)+\tilde{h}\left(j \mid \mathbf{x}_{t}, \Theta\right), j=1, \ldots, k_{\max },
$$

where the $I\left(j \mid \mathbf{x}_{t}, \Theta\right)$ is the indicator function defined in (6) . It is clear that this form meets the requirements of weight functions under the MWL framework. The rationale behind this form is that we give an award to the winning component, i.e., the $c$ th one, by assigning a weight whose value is larger than the corresponding $\tilde{h}\left(c \mid \mathbf{x}_{t}, \Theta\right)$. In contrast, we keep the weights of those rival components exactly equal to their corresponding $\tilde{h}\left(j \mid \mathbf{x}_{t}, \Theta\right)$ 's. That is, we give the winning component an award, but the rival ones not. Hence, this is actually another kind of award-penalization scheme. Such a scheme is able to make the genuine components survive in the learning process, whereas those "pseudo-clusters" will be faded out from the mixture gradually.

In (10), the new weight functions $\left\{\tilde{f}\left(1 \mid x_{l t}, \Phi\right), \tilde{f}\left(0 \mid x_{l t}, \Phi\right)\right\}$ should satisfy the following constraint:

$$
\lim _{h^{\prime}\left(i \mid x_{l t}, \Phi\right) \rightarrow 0} \tilde{f}\left(i \mid \mathbf{x}_{t}, \Theta\right) \log h^{\prime}\left(i \mid x_{l t}, \Phi\right)=0, i \in\{0,1\} .
$$




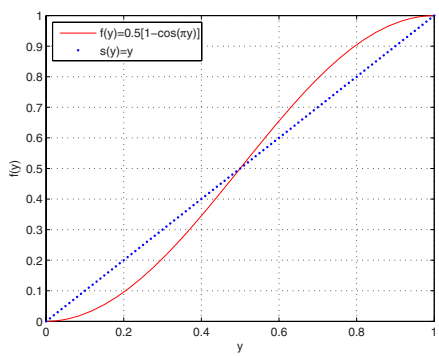

Fig. 1. $\mathrm{f}(\mathrm{y})$ vs. s(y). $\mathrm{f}(\mathrm{y})$ is plotted with "-"; $\mathrm{s}(\mathrm{y})$ is plotted with ".";

Accordingly, an applicable form is presented below:

$$
\tilde{f}\left(1 \mid x_{l t}, \Phi\right)=f\left(h^{\prime}\left(1 \mid x_{l t}, \Phi\right)\right), \tilde{f}\left(0 \mid x_{l t}, \Phi\right)=1-f\left(h^{\prime}\left(1 \mid x_{l t}, \Phi\right)\right),
$$

with

$$
f(y)=0.5[1-\cos (\pi y)], y \in[0,1] .
$$

$f(y)$ is plotted in Fig. 1, An interesting property of $f(y)$ can be observed from Fig. 1]

$$
y>f(y)>0, \text { for } 0<y<0.5 ; y<f(y)<1, \text { for } 0.5<y<1 .
$$

Hence, if $h^{\prime}\left(1 \mid x_{l t}, \Phi\right)>h^{\prime}\left(0 \mid x_{l t}, \Phi\right)$, it implies that the feature seems useful in the clustering. Subsequently, its log-likelihood $\log \left[w_{l} p\left(x_{l t} \mid \theta_{l j}\right)\right]$ is amplified by a larger coefficient $\tilde{f}\left(1 \mid x_{l t}, \Phi\right)$, meanwhile the log-likelihood of the "common" distribution is suppressed by a smaller coefficient $\tilde{f}\left(0 \mid x_{l t}, \Phi\right)$. A reverse assigning scheme is also held for the case when the feature seems less useful. In this sense, the function $f(y)$ rewards the important features that make the greater contribution to the likelihood, and penalizes those of insignificant features. Consequently, the estimation for the whole parameter set is given by:

$$
\hat{\Theta}_{M W L}=\arg \max _{\Theta}\left\{\tilde{Q}\left(\Theta, \mathbf{X}_{N}\right)\right\}
$$

An EM-like iterative updating by gradient ascent technique is used to estimate the parameter set. Algorithm 1 shows the pseudo-code description of the proposed algorithm. In implementation of this algorithm, $\left\{\alpha_{j}\right\}_{j=1}^{k}$ s must satisfy the constraint: $\sum_{j=1}^{k} \alpha_{j}=1$ and $0 \leq \alpha_{j}<1$. Hence, we also update $\left\{\beta_{j}\right\}_{j=1}^{k}$ s instead of $\left\{\alpha_{j}\right\}_{j=1}^{k}$ as shown in [6], and $\left\{\alpha_{j}\right\}_{j=1}^{k}$ are obtained by: $\alpha_{j}=e^{\beta_{j}} / \sum_{i=1}^{k} e^{\beta_{i}}, 1 \leq j \leq k$. As for another parameter $w_{l}$ with the constraint: $0 \leq w_{l} \leq 1$, we update $\gamma_{l}$ instead of $w_{l}$, and $w_{l}$ is obtained by the sigmoid function of $\gamma_{l}: w_{l}=\frac{1}{1+e^{-\tau \cdot \gamma_{l}}}, 1 \leq l \leq d$. The constant $\tau(\tau>0)$ is used to tune the shape of the sigmoid function. To implement a moderate sensitivity for $w_{l}$, i.e., an approximately equal increasing or decreasing quantity in both $\gamma_{l}$ and $w_{l}$, the slope of the sigmoid function around 0 with respect to (w.r.t.) $\gamma_{l}$ (namely, around 0.5 w.r.t. $w_{l}$ ) should be roughly equal to 1 . By rule of thumb, 


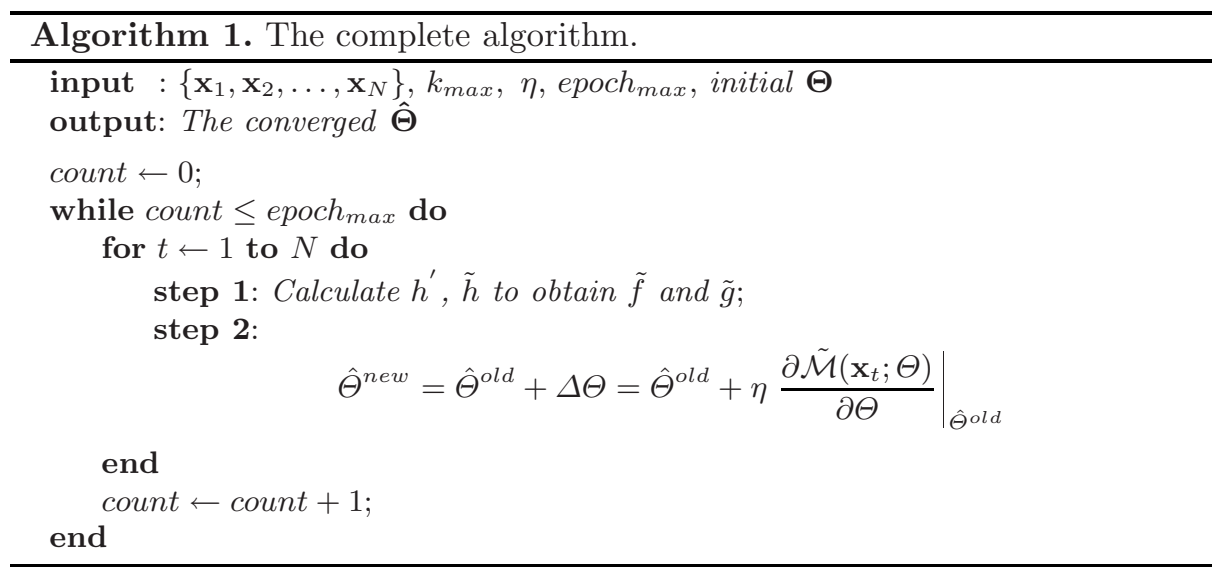

we find that a linear fitting of the two curves: $w_{l}=\frac{1}{1+e^{-\tau \cdot \gamma_{l}}}$ and $w_{l}=\gamma_{l}+0.5$ around 0 w.r.t $\gamma_{l}$ occurs around $\tau=4.5$. In the following, we will therefore set $\tau$ at 4.5 .

For each observation $\mathbf{x}_{t}$, the changes in the parameters set are calculated as:

$$
\begin{aligned}
& \Delta \beta_{j}=\left.\eta_{\beta} \frac{\partial \tilde{\mathcal{M}}\left(\mathbf{x}_{t} ; \Theta\right)}{\partial \beta_{j}}\right|_{\Theta^{\text {old }}}=\left.\eta_{\beta} \sum_{i=1}^{k_{\max }} \frac{\partial \tilde{\mathcal{M}}\left(\mathbf{x}_{t} ; \Theta\right)}{\partial \alpha_{i}} \cdot \frac{\partial \alpha_{i}}{\partial \beta_{j}}\right|_{\Theta^{o l d}} \\
& =\eta_{\beta}\left(\tilde{g}\left(j \mid \mathbf{x}_{t}, \Theta\right)-\alpha_{j}^{\text {old }}\right), \\
& \Delta m_{l j}=\left.\eta \frac{\partial \tilde{\mathcal{M}}\left(\mathbf{x}_{t} ; \Theta\right)}{\partial m_{l j}}\right|_{\Theta^{o l d}} \\
& =\eta \tilde{g}\left(j \mid \mathbf{x}_{t}, \Theta\right) \tilde{f}\left(1 \mid x_{l t}, \Phi\right) \frac{x_{l t}-m_{l j}^{\text {old }}}{\left(s_{l j}^{\text {old }}\right)^{2}}, \\
& \Delta s_{l j}=\left.\eta \frac{\partial \tilde{\mathcal{M}}\left(\mathbf{x}_{t} ; \Theta\right)}{\partial s_{l j}}\right|_{\Theta^{o l d}} \\
& =\eta \tilde{g}\left(j \mid \mathbf{x}_{t}, \Theta\right) \tilde{f}\left(1 \mid x_{l t}, \Phi\right)\left[\frac{\left(x_{l t}-m_{l j}^{\text {old }}\right)^{2}}{\left(s_{l j}^{\text {old }}\right)^{3}}-\frac{1}{s_{l j}^{\text {old }}}\right], \\
& \Delta c m_{l}=\left.\eta \frac{\partial \tilde{\mathcal{M}}\left(\mathbf{x}_{t} ; \Theta\right)}{\partial c m_{l}}\right|_{\Theta^{o l d}} \\
& =\eta \sum_{j=1}^{k_{\max }} \tilde{g}\left(j \mid \mathbf{x}_{t}, \Theta\right) \tilde{f}\left(0 \mid x_{l t}, \Phi\right) \frac{x_{l t}-c m_{l}^{\text {old }}}{\left(c s_{l}^{\text {old }}\right)^{2}}, \\
& \Delta c s_{l}=\left.\eta \frac{\partial \tilde{\mathcal{M}}\left(\mathbf{x}_{t} ; \Theta\right)}{\partial c s_{l}}\right|_{\Theta^{o l d}}
\end{aligned}
$$




$$
\begin{aligned}
& =\eta \sum_{j=1}^{k_{\max }} \tilde{g}\left(j \mid \mathbf{x}_{t}, \Theta\right) \tilde{f}\left(0 \mid x_{l t}, \Phi\right)\left[\frac{\left(x_{l t}-c m_{l}^{\text {old }}\right)^{2}}{\left(c s_{l}^{\text {old }}\right)^{3}}-\frac{1}{c s_{l}^{\text {old }}}\right] \\
\Delta \gamma_{l} & =\left.\eta \frac{\partial \tilde{\mathcal{M}}\left(\mathbf{x}_{t} ; \Theta\right)}{\partial \gamma_{l}}\right|_{\Theta^{\text {old }}}=\left.\eta \frac{\partial \tilde{\mathcal{M}}\left(\mathbf{x}_{t} ; \Theta\right)}{\partial w_{l}} \cdot \frac{\partial w_{l}}{\partial \gamma_{l}}\right|_{\Theta^{o l d}} \\
& =\eta \cdot \tau \cdot \sum_{j=1}^{k_{\max }} \tilde{g}\left(j \mid \mathbf{x}_{t}, \Theta\right)\left[\tilde{f}\left(1 \mid x_{l t}, \Phi\right)\left(1-w_{l}^{\text {old }}\right)-\tilde{f}\left(0 \mid x_{l t}, \Phi\right) w_{l}^{\text {old }}\right] .
\end{aligned}
$$

Generally speaking, the learning rate of $\beta_{j}$ s should be chosen as $\eta_{\beta}>\eta$ to help eliminate the much smaller $\alpha_{j}$ (we suggest $\eta=0.1 \eta_{\beta}$ ).

\section{Experimental Results}

\subsection{Synthetic Data}

We generated 1,000 2-dimensional data points from a Gaussian mixture of three components:

$$
0.3 * \mathcal{N}\left[\left(\begin{array}{l}
1.0 \\
1.0
\end{array}\right),\left(\begin{array}{ll}
0.15 & 0 \\
0 & 0.15
\end{array}\right)\right]+0.4 * \mathcal{N}\left[\left(\begin{array}{l}
1.0 \\
2.5
\end{array}\right),\left(\begin{array}{ll}
0.15 & 0 \\
0 & 0.15
\end{array}\right)\right]+0.3 * \mathcal{N}\left[\left(\begin{array}{l}
2.5 \\
2.5
\end{array}\right),\left(\begin{array}{ll}
0.15 & 0 \\
0 & 0.15
\end{array}\right)\right]
$$

In order to illustrate the ability of the proposed algorithm to perform automatic model selection and feature weighting jointly, we appended two additional features to the original set to yield a 4-dimensional one. The last two features are sampled independently from $\mathcal{N}\left(2 ; 2.5^{2}\right)$ as the Gaussian noise covering the entire data set. Apparently, the last two dimensions do not hold the same mixture structure, thus are not as significant as the first two dimensions in the partitioning process.

We initialized $k_{\max }$ to 15 , and all $\beta_{j}$ 's and $\gamma_{l}$ 's to 0 , which is equivalent to setting each $\alpha_{j}$ to $1 / 15$ and $w_{l}$ to 0.5 , the remaining parameters were randomly initialized. The learning rates are $\eta=10^{-5}, \eta_{\beta}=10^{-4}$. After 500 epochs, the mixing coefficients and feature weights are obtained by the proposed algorithm:

$$
\begin{aligned}
& \hat{\alpha}_{6}=0.4251 \hat{\alpha}_{8}=0.2893 \hat{\alpha}_{15}=0.2856 \hat{\alpha}_{j}=0, j \neq 6,8,15 \\
& \hat{w}_{1}=0.9968 \hat{w}_{2}=0.9964 \hat{w}_{3}=0.0033 \hat{w}_{4}=0.0036 .
\end{aligned}
$$

The feature weights of the first two dimensions converge close to 1 , while those of the last two dimensions are assigned close to 0 . It can be seen that the algorithm has accurately detected the underlying cluster structures in the first two dimensions, and meanwhile the appropriate model order and component parameters have been well estimated.

\subsection{Real-World Data}

We further investigated the performance of our proposed algorithm on serval benchmark databases [7] for data mining. The partitional accuracy of the algorithm without the prior knowledge of the underground class labels and the 
Table 1. Results of the 30-fold runs on the test sets for each algorithm, where each data set has $N$ data points with $d$ features from $k^{*}$ classes

\begin{tabular}{|c|c|c|c|}
\hline Data Set & Method & $\begin{array}{c}\text { Model Order } \\
\text { Mean } \pm \text { Std }\end{array}$ & $\begin{array}{c}\text { Error Rate } \\
\text { Mean } \pm \text { Std }\end{array}$ \\
\hline wine & RPEM & $2.5 \pm 0.7$ & $0.0843 \pm 0.0261$ \\
$d=13$ & EMFW & $3.3 \pm 1.4$ & $0.0673 \pm 0.0286$ \\
$N=178$ & NFW & $2.8 \pm 0.7$ & $0.0955 \pm 0.0186$ \\
$k^{*}=3$ & proposed method & $3.3 \pm 0.4$ & $\mathbf{0 . 0 2 9 2} \pm \mathbf{0 . 0 1 4 5}$ \\
\hline heart & RPEM & $1.7 \pm 0.1$ & $0.3167 \pm 0.0526$ \\
$d=13$ & EMFW & $2.5 \pm 0.5$ & $0.2958 \pm 0.0936$ \\
$N=270$ & NFW & $2.2 \pm 0.4$ & $0.2162 \pm 0.0473$ \\
$k^{*}=2$ & proposed method & fixed at 2 & $\mathbf{0 . 2 0 4 2} \pm \mathbf{0 . 0 3 7 9}$ \\
\hline wdbc & RPEM & $1.7 \pm 0.4$ & $0.2610 \pm 0.0781$ \\
$d=30$ & EMFW & $6.0 \pm 0.7$ & $0.0939 \pm 0.0349$ \\
$N=569$ & NFW & $3.0 \pm 0.8$ & $0.4871 \pm 0.2312$ \\
$k^{*}=2$ & proposed method & $2.6 \pm 0.6$ & $\mathbf{0 . 0 8 3 4} \pm \mathbf{0 . 0 3 8 6}$ \\
\hline ionosphere & RPEM & $1.8 \pm 0.5$ & $0.4056 \pm 0.0121$ \\
$d=34$ & EMFW & $3.2 \pm 0.6$ & $0.1968 \pm 0.0386$ \\
$N=351$ & NFW & $2.2 \pm 0.5$ & $0.3201 \pm 0.0375$ \\
$k^{*}=2$ & proposed method & $2.9 \pm 0.7$ & $0.2029 \pm 0.0667$ \\
\hline
\end{tabular}

relevancy of each features were measured by the error rate index. We randomly split those raw data sets into equal size for the training sets and the testing sets. The process was repeated 30 times, yielding 30 pairs of different training and test sets. For comparison, we conducted the proposed algorithm, the RPEM algorithm, as well as the approach in [5] (denoted as EMFW). To examine the efficacy of the feature weight function $f\left(. \mid x_{l t}, \Phi\right)$, we also conducted the algorithm (denoted as NFW) with the feature weight function in (12) setting to $s(y)=y, y \in[0,1]$, i.e., no penalization on the lower level Gaussian mixture in feature relevancy estimation. The means and standard deviations of the results obtained on the four sets are summarized in Table 1] from which we have the three remarks as follows:

Table 2. The average weighting results of the 30 fold-runs on the real data, where the feature weights for $w d b c$ and ionosphere are not included as the number of their features is too large to accommodate in this Table

\begin{tabular}{|c|c|c|c|c|c|c|c|c|c|c|c|c|c|}
\hline \multirow{2}{*}{ Data set } & \multicolumn{13}{|c|}{ Feature } \\
\hline & 1 & 2 & 3 & 4 & 5 & 6 & 7 & 8 & 9 & 10 & 11 & 12 & 13 \\
\hline wine & 0.9990 & 0.8799 & 0.0256 & 0.2831 & 0.2354 & 0.9990 & 0.9990 & 0.0010 & 0.9900 & 0.9869 & 0.7613 & 0.9990 & 0.0451 \\
\hline heart & 0.0010 & 0.6776 & 0.5872 & 0.0010 & 0.0010 & 0.9332 & 0.5059 & 0.0010 & 0.8405 & 0.3880 & 0.3437 & 0.4998 & 0.7856 \\
\hline
\end{tabular}

Remark 1: In Table 1 it is noted that the proposed method has much lower error rates than the RPEM algorithm that is unable to perform the feature discrimination. Table 2 lists the mean feature weights of the sets obtained by the proposed algorithm in the 30 runs, indicating that only several features have good discriminating power. Remark 2: Without the feature weight functions to assign unequal emphases on the likelihood for the lower level Gaussian mixture, it is found that the performance of the NFW algorithm is unstable because of no enough penalization to the model complexity. It therefore validates the design of the proposed weight functions for weighting features. Remark 3: It is observed 
that the method in [5] tends to use more components for the mixture, whereas the proposed algorithm not only gives general lower mismatch degrees, but also produces much more parsimonious models.

\section{Conclusion}

In this paper, a novel approach to tackle the two challenges for Gaussian mixture clustering, has been proposed under the Maximum Weighted Likelihood learning framework. The model order for the mixture model and the feature weighting are obtained simultaneously and automatically. Experimental results have shown the promising performance of the proposed algorithm on both the synthetic and the real-world data sets.

\section{References}

1. Liu, H., Yu, L.: Toward integrating feature selection algorithms for classification and clustering. IEEE Transactions on Knowledge and Data Engineering 17, 491$502(2005)$

2. Dash, M.C., Scheuermann, K., Liu, P.H.: Feature selection for clustering-A filter solution. In: Proceedings of 2nd IEEE International Conference on Data Mining, pp. 115-122. IEEE Computer Society Press, Los Alamitos (2002)

3. Dy, J., Brodley, C.: Feature selection for unsupervised learning. Joural of Machine Learning Research 5, 845-889 (2004)

4. Figueiredo, M.A.T., Jain, A.K., Law, M.H.C.: A feature selection wrapper for mixtures. LNCS, vol. 1642, pp. 229-237. Springer, Heidelberg (2003)

5. Law, M.H.C., Figueiredo, M.A.T., Jain, A.K.: Simultaneous feature selection and clustering using mixture models. IEEE Transactions on Pattern Analysis and Machine Intelligence 26, 1154-1166 (2004)

6. Cheung, Y.M.: Maximum weighted likelihood via rival penalized EM for density mixture clustering with automatic model selection. IEEE Transactions on Knowledge and Data Engineering 17, 750-761 (2005)

7. Blake, C.L., Merz, C.J.: UCI Repository of machine learning databases (1998), http://www.ics.uci.edu/mlearn/MLRepository.html 